\title{
New Architecture of Network Elements
}

\author{
Vlad is lav Skorpil, Martin Kral \\ Department of Telecommunications, \\ Brno University of Technology, \\ Purkynova 118, 612 00 Brno, Czech Republic \\ skorpilafeec.vutbr.cz,
}

\begin{abstract}
This paper describes design and computer simulation of a new architecture of a node active network element, based on artificial neural network technology with the support of priority processing for different connection types. As an example of a network element was selected switching area. This network element with optimized switching area is able to transfer large data quantity with minimum delay. Architecture of network element, that contains artificial neural network for optimized priority switching is described in this paper. It describes implementation of neural network in control process for data units switching. The programming language MATLAB 7.0 was used for software simulation. Network elements with new architecture, which uses a neural network, as well as intimated simulation, are suitable for working for example in personal wireless network communication systems.
\end{abstract}

Keywords: Neural network, Switch, Switching area, Network element, Neuron

\section{Introduction}

The switching-over is the basic function of the active network elements that work above physical layer of OSI model. The function of switch consists at that, so data incoming on inputs must be transported to target output and it what fastest. The speed can be limited by blocking, it is caused by situation when data flow from two or more inputs directs to one output. It is necessary in this case for everyone data flow to reserve fair output allocation. Basic communication protocols for computer networks, like protocol IP or Ethernet, does not contain any mechanism for controlling of fair or priority communication channel reserving and they use queue of FIFO type. As modern multimedia applications would such mechanis $m$ often need, the poss ibilities how to guarantee this mechanism are searched very hardly in present. The common quality of these solutions is, that it is assumed the identification of single data or groups of data flows and priority allocation, on bas is that the switching process is made. 


\section{Model of the switch making use of artificial neural network}

The basic model of switch-over switching area consists of several partial b locks. The block diagram of the switching area is illustrated on the Fig.1. The received data units are first storage into input buffer. As data units contain target address, it is possible to determine over which output port they leave switching system. Except information about target port are detected also information about data flow whereto the data unit belongs to. Mentioned information are assembled from of all data units retained in input buffers and serve as input data for neural network.

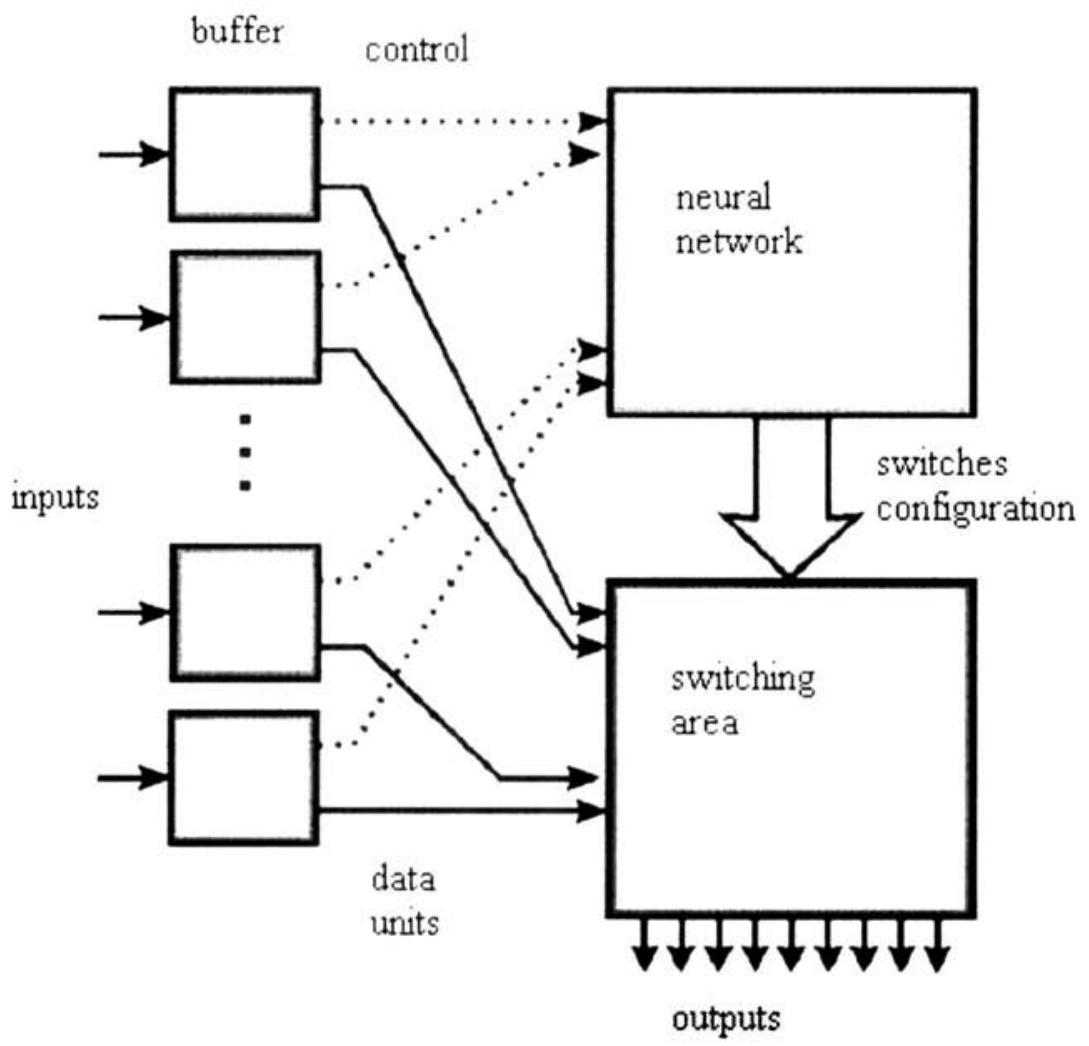

Fig. 1 Block diagram of switching area utilizing neural network

In the event of, so the frames in the port's buffer have such target addresses, so to everyone's output direction exists at most one requirement, it is possible directly put together N-dimensional vector, formed from priorities of single requirements. The first component of the vector includes the frame's priority oriented to the first output, the second one includes the frame's priority oriented to the second output and so one. 
The vector must always contain concrete values. In the event of, so no requirement is to output port, have to be on relevant position of the vector the value corres ponding to the lowest priority.

In the event of, so more frames in buffer are headed to the same output and it either with the same or with the different priority, have to exist way how to determine, which concrete one will be chosen for optimization process. In the event of different priority the frame with the highest priority is chosen of course. As far as the data units have the same priority, another algorith $\mathrm{m}$ for selection must be implemented.

It is possible from vectors of priority since single input ports to create the $\mathrm{NxN}$ dimensional matrix, that shall contain input conditions, to be necessary for optimize. Like answer on this input data the neural network will generate explicit configuration of switches, on basis that the switches in the switching area will be set. Generated configuration will be in the form of $\mathrm{NxN}$ dimensional matrix, so-called configuration matrix. Demand on generated result is to be connected always one input and one output. This condition will be projected to the configuration matrix that is generated by neural network thus, that at every row and at every column will be exactly one active output, i.e. the value is one. The other will be non-active, i.e. the value is zero. Past setting of switches it can come to own transmission of data units from input buffer units to outputs of system

It was already states, that setting of the switches has to be optimal in light of the matrix of priority. To the configuration matrix, obtained as a result that is generated by the neural network, we can look like to a template. By the help of this template, it is possible to choose elements from arbitrary $\mathrm{NxN}$ dimensional matrix. $\mathbf{P}$ so, that from the matrix $\mathbf{P}$ are selected only elements, which stands on the position, where the configuration matrix has the value of one. That way we obtain from the matrix $\mathbf{P} \mathbf{N}$ selected elements by the help of the template. When we apply this template to the matrix of priority obtained from the input buffers, we are able to determine the priority of elements, that just will be transported. We can then formulate the optimization exercise so, in order to be summation of priorities minimal. But at the same time, how it was already noted above, we have to guarantee in every row and column of matrix only one value of one. This condition is not completely accorded to the optimization travelling-salesman problem, but it is very close to it.

\section{The basic scheme of the element}

We think over the single-stage switching area, which has three inputs and three outputs, it is switch on the Fig.2. The switching area is realized on the cross-bar switch, i.e. in the described case the switching area with 9 switching points. We can connect arbitrary input to arbitrary output. However in one time moment $t$ we can transfer information from one input only to one output in terms of only one's switch. 


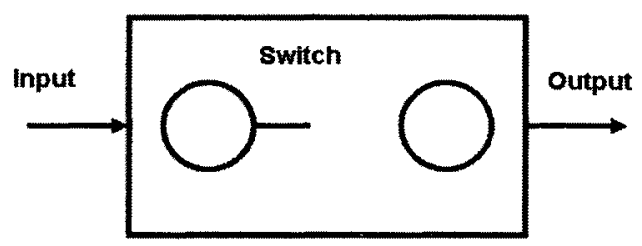

Fig.2 Switch

We create the switching area by in a way, that the switches will be shape partial positions, generally $\mathrm{m}-\mathrm{n}$ dimensional matrix, in described case $3 \times 3$ dimensional one. We do not speculate about mult iplexing of inputs or outputs, every switching point of the switching area presents one switch (Fig.3). The multiplexing has meaning for generally $\mathrm{m}-\mathrm{n}$ dimensional switching area, main benefits have economic character, saving of a material and of a place.

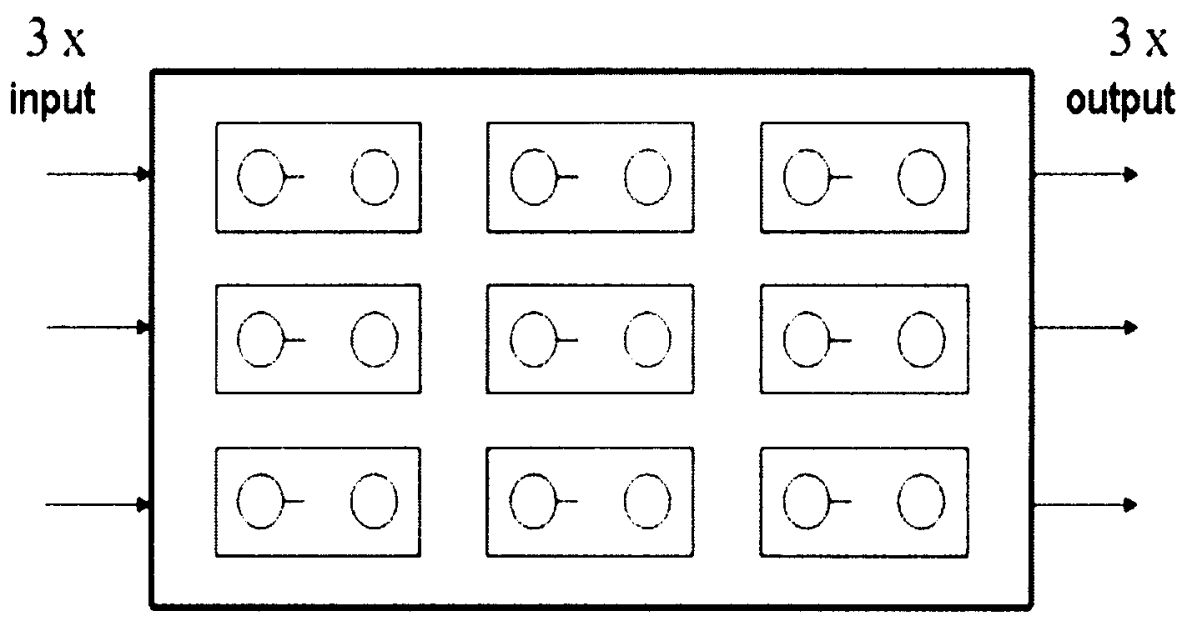

Fig.3 Switching area

The function of switching area is, in order to data unit, which reveals on the input, was transported to relevant output of the area and continues further to a receiver. As far as we would introduce data units into switching area only from one input and other inputs would be cancelled, function of all a rea would be greatly unused and efficiency would be small. That is why we want, to appear in the same instant of time data units on every input, which have to be transported pass through switching area. One quite serious problem is here. It can become, so on two and more inputs in the same time moment data units appear, which are addressed to the same output. Then the question 
arrives, which one of data have priority of processing and for which of them is not real-time transmission critical. This question is solved by the priority processing method.

We return back to the switching area. We introduce the simplest network about so much message sources (data), how much it has inputs in the node and so much receivers, how much it has outputs. We should have only one node in the network. So that we prove to deliver the right data units to the correct receiver, we have to set up addressing. The simple two-bits addressing word, how in the event of input, that way also of output of the switching area, is used in our example (Fig.4). The first input has address 01 , the second one 10 and the third one 11 . The first output has address 01 , the second one 10 and the third one 11 . The resolution, whether or not it walks about input or output address, is given by virtue of the data frame header, where it is stated, whether it walks about source or target address. The priority is stated also in the header. The frame structure is on the Fig.5,

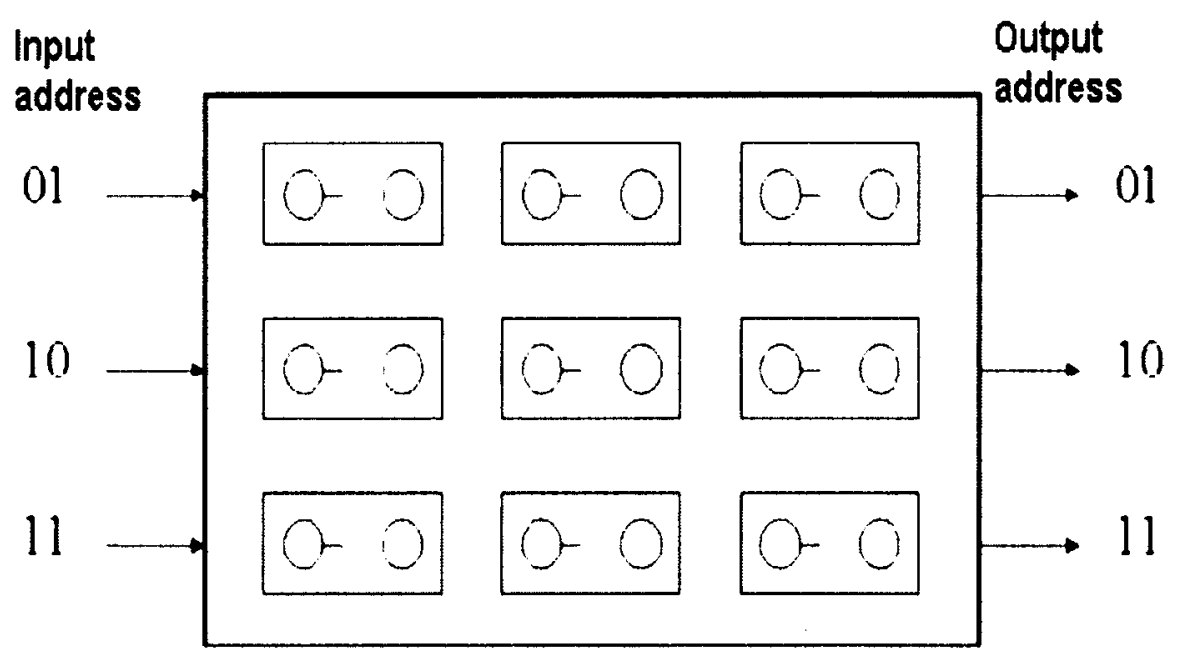

Fig.4 Switching area with addressing

\begin{tabular}{|c|c|c|c|}
\hline $\begin{array}{c}2 \text { bits } \\
\text { Target address }\end{array}$ & $\begin{array}{c}2 \text { bits } \\
\text { Source address }\end{array}$ & 4 bits Priority & 16 bits Data \\
\hline
\end{tabular}

Fig.5 Frame structure 


\section{Control of switching area}

The switching area would not be operational without control. The control is here realized by control matrix, which has dimension of switching area and every element of this matrix control one switching point. It is matrix with the dimension $3 \times 3$, in described case.

$$
\mathbf{C}=\left(\begin{array}{lll}
x & x & x \\
x & x & x \\
x & x & x
\end{array}\right)
$$

Indexing of switching area according to control matrix $\mathbf{C}$ is simple. The switching point, which switches the first input, i.e. with the address 01 , to the first output (address 01) corresponds to the matrix element with the index $11\left(\mathrm{C}_{11}\right)$. And farther, the switching point which switches the first input to the second output will be operating by the element of control matrix with the index $12\left(\mathrm{C}_{12}\right)$, etc. The elements of the matrix put on binary value 0,1 . The value of logical 1 means bracing of the switching point and thereby the way through the switching area is closed and data units from the appropriate input are transported to the given output. The switching point, controlled by the appropriate element of matrix about the value of logical 0 , will be in a sleep position, the switch does not close and it means, so the data units from appropriate input were routed to other output. On the Fig. 6 we can see the particular graphic example. The outputs of switching area are for simpler notion removed from the right side to low one, because on the original picture is not clear, which switching point corresponds to which output. The control matrix will be given by elements:

$$
\mathbf{C}=\left(\begin{array}{lll}
0 & 1 & 0 \\
1 & 0 & 0 \\
0 & 0 & 1
\end{array}\right)
$$

It is given, at this structure of control matrix, so logical value 1 of the matrix element $\mathrm{C}_{12}$ switches switching point, which connects the first input to the second output. Further the logical value 1 of the matrix element $C_{21}$ switches switching point which connects the second with the first output and last the logical value 1 of the matrix element $\mathrm{C}_{33}$ switches switching point which connects the third input with the third output. 


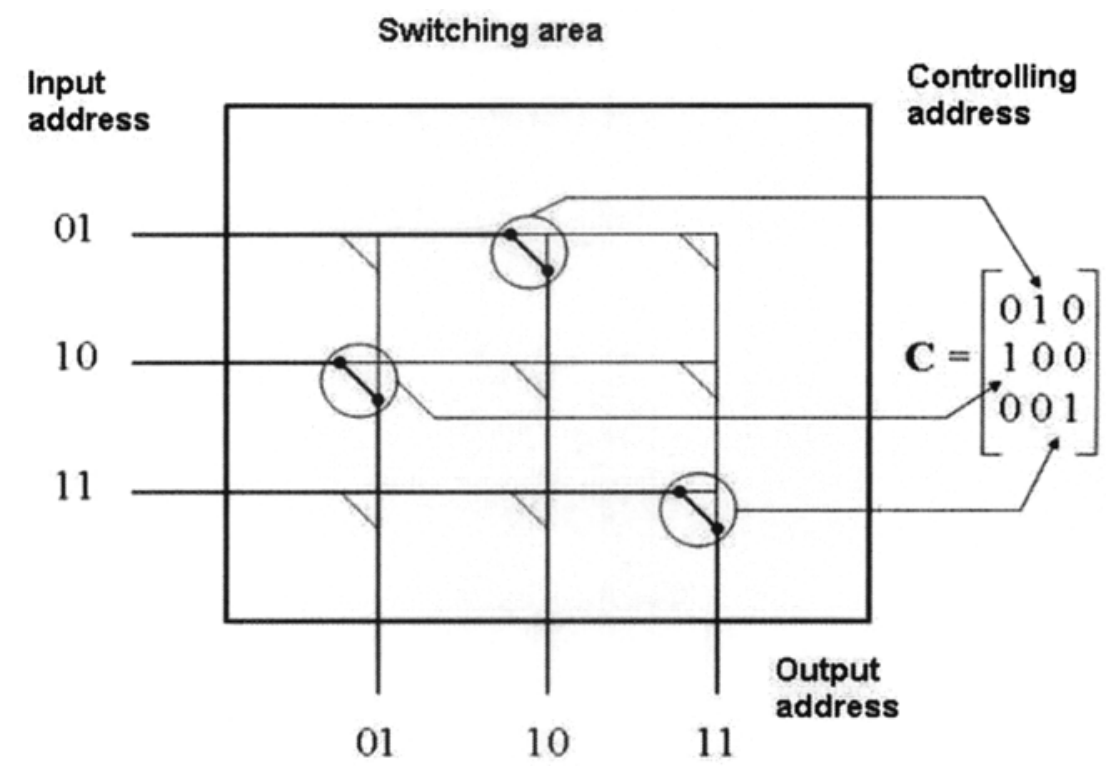

Fig.6 Switching area controlled by control matrix

\section{Scheme of neurons examining priority selection}

The most important part of the whole network element is a block, which examines priority selection of frames. This frames will be subsequently sent to the switching area. The block by virtue of certain information evaluates the most acceptable or rather the most important frame from the possible selection and gives up advice for sending it. Just this block works on the principle of neural network. The neurons practise this important priority selection. The scheme of neurons examining priority selection in on the Fig. 7 . 


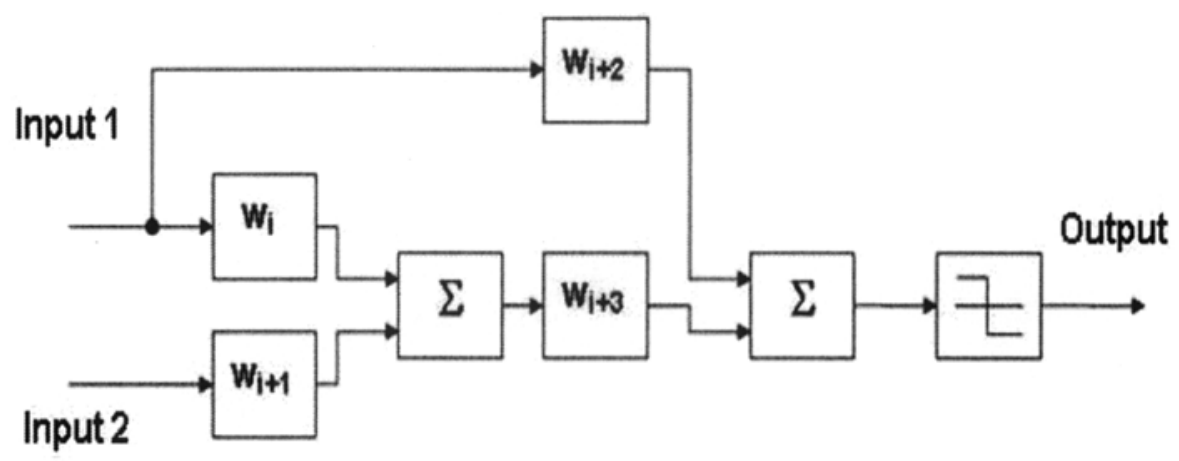

Fig.7 Scheme of neurons examining priority selection

The block, that examines priority selection, contains from two neurons. This block selects within one output of switching area if you like of one output address. For our actual switching area, and consequently 3 possible output addresses, 3 these blocks will be used. The values of priority of single frames, which ask for input to the switching area, are set to the input of the block. The frames have one sharing, all frames have the same final address as well as different source address. It comes to this, that frames from different directions are routing to the one particular output. So that we decide, which frame to choose, we have to compare their priority. At the beginning we take priority from frames, which are on the first two inputs, during which time the priority of frame, which applies approach to the first input of switching area, will allocate to the input 1 . The first neuron these two priority sums and numbered value sends to the second input of the second neuron. On the first input of the second neuron we send priority from the input 1 . The result is sent to the block of non-linear function after numbered of both values to the summarize block of the second neuron. It decides, whether on the output will be value $\log 0$ or $\log 1$ and all process repeats. The value of output controls partly the assignment of priority to the inputs one and two as well as the associated matrix of priority $\mathbf{B}$.

\section{Conclusion}

The aim of this research was assembling of theoretical materials and knowledge concerning to the problems of network elements and artificial neural networks and then design and subsequently computer simulation of a new architecture of node active network element. This network element will be founded on the technology of artificial neural networks and will support priority processing of different types of 
connection. The optimization of switching area of these active elements is very actual. In the paper is stated the architecture of network element, which includes neural network for optimizing of priority switching. It was used programme language MATLAB 7.0 for the software simulation. The idea of the implementation of neural network at control process of switching of data units is indicated at simulation. It is also during the simulation demonstrated, to what purpose is every bloc in the element used.

At last it is possible to claim, so target of the research were realized. It was designed a simulated network element, containing in the process of control of switching area artificial neural network. The element further switches single data units making provision for priority. The next research will be expansion of the number of inputs and outputs of the network element.

Acknowedgement. This research was supported by the grants:

No 102/07/1503 Advanced Optimizing the design of Communication Systems via Neural Networks. The Grant Agency of the Czech Republic (GACR)

Grant 1884/2007/F 1/a "Innovation of computer networks partic ipation in high-speed communication" (grant of the Czech Ministry of Education, Youth and Sports)

Grant 1889/2007/F 1/a ", Repair of digital exchanges education in the course Access and Transport Networks" (grant of the Czech Ministry of Education, Youth and Sports)

No MSM 0021630513 Research design of Brno University of Technology" Electronic commun ication systems and new generation of technology (ELKOM)"

2E06034 Lifetime education and professional preparation in the field of telematics, teleinformatics and transport telematics (grant of the Czech Ministry of Education, Youth and Sports)

\section{References}

1. Gupta,M. and LiangJ. Static and dynamic neural network, John Wiley \& Sons, Inc., New Jersey, 2003

2. Jirsik, V. and Hráček,P. Neural networks, experts systems and speech recognition., BUT Brno 2004

3. Novák,M. Artificial near al networks and applications. C. H. Beck, Praha, 1998

4. Martán, P. Visualization of selected methods of machine learning MU, 2002

5. Novotmý, V. Network architecture. BUT, Brno, 2002

6. Molnár,K. Aplication of neural networks in high-speed active network elements. BUT, Brno, 2002

7. Minsky,M. and Papert, S. Perceptrons, MIT Press, 1969

8. Hassoun,M.H. Fundamentals of artifical neural network, MT Press, 1995

9. Síma J. and Neruda, R. Theoretical questions of neural networks. Maty zpress, Praha, 1996

10. Fausett,L. Fundamentals of Neural Networks, Architecture, Algorithms and Applications, Prentice Hall, 1994 
11. Kosko,B. Neural Networks and Fuzzy Systems, Prentice-Hall International, Inc., London, 1992

12. Molnar,K. Switching area of ATM switch controlled by neural network. BUT, Brno 1999

13. Rinipe, J. and Euliano, R.N. and Lefebvre, W.C. Neural and Adaptive Systems, John Wiley \& Sons, Inc., USA, 1999

14. Krbilová, 1. and Vestenický, P. Use of Intelligent Network Services in Proc. of ITS. RTT, CTU Prague, 2004

15. Král,M. Design of new network element architecture. BUT, Brno 2006

16. Vestenický, M. and Vestenický, P. Evolutionary Algorithms in Design of Switched Capacitors Circuit in Proc. of International Workshop „Digital Technologies 2004“. Slovak Electrical Society and University of Zilina, pp.34-37, 2004

17. Vestenický,P. The Prediction Properties of Kalman Filter in Proceedings of TRANSCOM '95. UT\&C, Zilina, pp. 243-248, 1995

18. Krbilová, I. and Vestenický,P.: Forgalomszabályozásés szolgáltatásminöség" Magyar távközlés 7, Vol.. 6/96, pp. 32-33, 1996

19. Bubeniková, E. and Vestenický, P. Princip les Of The Intranet Information Sy stem Creation in Proc. of ELEKTRO'99 Conference Proceedings, section Information \& Safety Systems. University of Žilina, pp. 77-81, 1999

20. Vestenický, P. The Functions of ATM Interfaces in Proc. of DDECS ' 97 Conference Proceedings. VSB Technical Univ ersity, Ostrava, pp. 186-191, 1997

21. Vestenický, P. Optimization of Selected RFID System Parameters in Proc. of. AEEE 3, Vol. 2, pp. 113-114, 2004 